\title{
latrogenic Thyrotoxicosis Secondary to Compounded Liothyronine
}

\author{
Ajay Bains, Ami-Joseph Brosseau, and David Harrison
}

\section{INTRODUCTION}

$\mathrm{T}$ 'his article describes 2 patients* who were admitted to the Royal Jubilee Hospital in Victoria, British Columbia, with different presenting illnesses but similar laboratory values, similar medication histories, and the same final diagnosis. Both patients had received the same compounded formulation of liothyronine, which had resulted in thyrotoxicosis.

\section{CASE REPORTS}

\section{Case 1}

A 71-year-old woman presented with ST segment elevation myocardial infarction (STEMI) associated with hypotension (100/70 mm Hg) and dizziness. Her husband stated that she appeared confused and was experiencing visual hallucinations. Her medical history was significant for hypertension, pulmonary embolism, hypothyroidism, and gastroesophageal reflux disease. Preadmission medications were reported as amlodipine $2.5 \mathrm{mg}$ daily and pantoprazole $40 \mathrm{mg}$ daily. She denied any recreational drug use or history of mental illness (including hallucinations). She was living independently at home with her husband.

The patient's atypical cardiac symptoms prompted referral to the neurology service. However, neurogenic causes such as Hashimoto encephalitis and cerebrovascular accident were ruled out by negative results on thyroid antibody testing and computed tomography of the head. Incidentally, the laboratory panel for Hashimoto encephalitis included measurement of thyroid hormones, which showed that the level of free tri-iodothyronine was greater than $50 \mathrm{pmol} / \mathrm{L}$ (normal range 3-6.8 pmol/L). Thyroidstimulating hormone was $0.03 \mathrm{mIU} / \mathrm{L}$ (normal range 0.34-5.6 $\mathrm{mIU} / \mathrm{L}$ ) and free thyroxine was $15 \mathrm{pmol} / \mathrm{L}$ (normal range

*The authors obtained consent from the patients and from Island Health Authority (the regional health authority where they received care) for publication of these case reports.
10-23 pmol/L). The thyroid was not examined, and Grave disease was ruled out on the basis of the normal thyroxine level.

In light of the laboratory results, a more detailed investigation for an exogenous iatrogenic cause was initiated. The only discrepancy between the patient's in-hospital medications and her preadmission medications was a compounded product that she had been taking before the admission, specifically compounded liothyronine capsules $(25 \mu \mathrm{g}$ of liothyronine in hydroxypropyl methylcellulose [hypromellose] for once-daily dosing). The diagnosis was iatrogenic thyrotoxicosis secondary to a compounding error involving liothyronine. The patient's symptoms, her history related to the current illness, and the supratherapeutic level of tri-iodothyronine were consistent with acute toxicity. She was given supportive care until her mental status and hemodynamics returned to normal and was then discharged to home. The consequences of the poisoning included STEMI, acute renal injury secondary to hypotension (with serum creatinine twice her baseline value), and a 13-day hospital stay for delirium. Hospital staff contacted the patient's outpatient pharmacy and alerted them to these findings.

\section{Case 2}

A 59-year-old woman presented with 2 days of intractable vomiting upon returning from a trip to Hawaii. Her medical history was significant for hypothyroidism, hypertension, type 2 diabetes mellitus, and cholecystectomy. The provincial PharmaNet database indicated that the patient's medications before admission were perindopril $4 \mathrm{mg}$ daily, metformin $500 \mathrm{mg}$ 3 times daily, acetylsalicylic acid $81 \mathrm{mg}$ once daily, and an unspecified compounded preparation. The patient had been taking these medications (except for the compounded preparation) for more than 2 years, and no drug-related problems were identified that would have caused the presenting symptoms. She lived independently at home with her husband and denied recreational drug use. Despite negative results on work-up for infectious causes of her vomiting, she continued to feel unwell. 
Laboratory investigations showed hypernatremia (sodium $151 \mathrm{mmol} / \mathrm{L}$ [normal range $135-145 \mathrm{mmol} / \mathrm{L}]$ ) and a hyperosmolar state (serum osmolality $330 \mathrm{mOsm} / \mathrm{kg}$ [normal range 280-300 mOsm/kg]). As the admission progressed, she continued to experience electrolyte abnormalities, specifically hypernatremia (sodium $151 \mathrm{mmol} / \mathrm{L}$ ) and hypokalemia (potassium $3.1 \mathrm{mmol} / \mathrm{L}$ [normal range $3.5-5 \mathrm{mmol} / \mathrm{L}$ ]). In addition, delirium started to develop within 3 days of her admission to hospital. A detailed medication history was taken, with the patient's husband as a collateral source of information. Her husband stated that she had been compliant with all of her medications except compounded liothyronine capsules $(25 \mu \mathrm{g}$ of liothyronine in hypromellose once daily). He noted that she had been unable to fill the prescription for compounded liothyronine before leaving on the trip to Hawaii, but she had initiated the compounded thyroid medication recently, upon returning home from the trip.

A thyroid panel yielded the following results: thyroidstimulating hormone $0.2 \mathrm{mIU} / \mathrm{L}$ (below normal), free thyroxine $16 \mathrm{pmol} / \mathrm{L}$ (within normal range), and tri-iodothyronine $39 \mathrm{pmol} / \mathrm{L}$ (above normal). The recent re-initiation of the compounded liothyronine preparation combined with these laboratory values indicated acute toxicity. Iatrogenic liothyronine poisoning was diagnosed, and the patient was given supportive care. She was discharged once she stopped vomiting and her electrolytes were within normal range (after a 10-day hospital stay).

\section{DISCUSSION}

About $95 \%$ of oral liothyronine is absorbed from the gastrointestinal tract within $4 \mathrm{~h}$ of administration, and this drug has a biological half-life of about 2.5 days. ${ }^{1}$ The onset of action is within $2-3 \mathrm{~h} .{ }^{1}$ Supraphysiological levels can be achieved within $6 \mathrm{~h}$ after an oral dose, and the level declines to the normal range within $24 \mathrm{~h}^{2}$ Liothyronine is the main pharmacologically active agent responsible for increases in cellular metabolism (with thyroxine being deiodinated in the periphery to produce liothyronine). This process in turn causes adrenergic stimulation, resulting in manifestations of pathologic, neurologic, gastrointestinal, and cardiovascular symptoms. ${ }^{2}$ According to the British Columbia Drug and Poison Information Centre, ${ }^{3}$ acute overdose usually results in only minor symptomatic discomfort. More severe intentional or unintentional overdose has resulted in thyrotoxicosis several days after ingestion, as experienced by the 2 patients described above. The toxic effects experienced by these patients can likely be explained by excess stimulation of the nervous, gastrointestinal, and cardiovascular systems by the toxic doses of liothyronine.

Both of these patients came under the care of the clinical teaching unit at the Royal Jubilee Hospital within 2 days of admission. The clinical pharmacist (A.B.) and the medical team contacted the community pharmacy of both patients, which (not coincidentally) was the same compounding pharmacy. This further solidified the diagnosis of iatrogenic liothyronine poisoning secondary to a compounding error involving excess dosage. The compounding pharmacy stated that these were the only 2 patients for whom it had recently prepared compounded liothyronine, and this type of error was plausible. The compounded product itself was not analyzed because the clinical team felt that the 2 patients sharing the same compounding pharmacy and the same diagnosis was strong enough evidence of incorrect compounding. Both patients returned the medication to the pharmacy and filed a complaint.

These 2 case reports raise specific concerns about liothyronine replacement, as well as general concerns about compounded medications. A typical dose for liothyronine is between 5 and 25 $\mu \mathrm{g} /$ day (maximum $150 \mu \mathrm{g} /$ day). ${ }^{1}$ Although a commercial product is available (Cytomel), prescribers for both of the patients described above had insisted that the liothyronine be compounded, on the premise that the drug has a longer duration of action when mixed with hypromellose. ${ }^{4}$ The excess dosage error arose through a process called geometric dilution. This process is employed to work around the inability of pharmacies to measure out medications in microgram quantities. Because the amount of product required in each dose is usually between 5 and $25 \mu \mathrm{g}$, compounding pharmacies must start the process by weighing out 10 times the desired amount and diluting it with an inert agent (usually lactose or hypromellose). This mixture is then put into carefully weighed capsules. ${ }^{5}$ If there is a measuring error with either the diluent or the active drug, each capsule could contain 10 to 100 times the intended dose. The clinical pharmacist contacted the pharmacy in question and asked that the preparation be recalled and that compounding procedures be audited.

The 5th edition of the Poison Management Manual of the British Columbia Drug and Poison Information Centre ${ }^{3}$ reported a case in which a patient received 1000 times the intended dose of liothyronine and experienced symptoms similar to those described here. The report mentioned that an unintended dosing error had occurred, but did not go into detail as to how the error occurred. ${ }^{3}$ To the current authors' knowledge, incidents like those described in this article are rare, but they do raise concerns about compounded medications and compounding techniques. Furthermore, they raise concerns about whether patient safety is compromised when a prescriber elects not to use a commercially manufactured product in favour of a compounded preparation. In this situation, the patient is at greater risk of a medication error for 2 reasons. The first, as outlined above, relates to errors in preparation and quality control. The second, at least in the province of British Columbia, is that the medication will be entered in the provincial pharmaceutical database (the $\mathrm{BC}$ PharmaNet system) only as a "compounded preparation", without specification of the contents of the preparation. Such ambiguity can then be clarified only through discussion with the patient directly or the patient's retail pharmacy. 


\section{References}

1. Cytomel product monograph. Kirkland (QC): Pfizer Canada Inc; 2012 Oct 16.

2. Sypniewski E. Comparative pharmacology of the thyroid hormones. Ann Thorac Surg. 1993:56(1 Suppl):S2-6; discussion S6-8.

3. Cadario BJ, Leathem AM, editors. Poison management manual. 5th ed. Vancouver (BC): British Columbia Drug and Poison Information Centre; 2003. p. 426-33.

4. Li CL, Martini LG, Ford JL, Roberts M. The use of hypromellose in oral drug delivery. J Pharm Pharmacol. 2005:57(5):533-46.

5. Timmons ED, Timmons SP. Custom-compounded micronized hormones in a slow-release capsule matrix. Int J Pharm Compound. 2002:6(5):378-9.
Ajay Bains, BSC(Pharm), ACPR, BCPS, is with the Pharmacy Department, Island Health Authority, Victoria, British Columbia.

Ami-Joseph Brosseau, BA, MD, is with the Island Medical ProgramFamily Practice, Victoria, British Columbia.

David Harrison, BSC, MSc, MD, is with the Island Medical ProgramFamily Practice, Victoria, British Columbia.

Competing interests: None declared.

\section{Address correspondence to:}

Ajay Bains

Pharmacy Department

Island Health Authority

PO Box 31082 University Heights

Victoria BC V8N 6J3

e-mail: ajaybains@ajaybains.com

Acknowledgement: The authors would like to acknowledge Michelle Bains for assistance in preparation of the manuscript.

\section{CJHP Call for photographs}

The Canadian Journal of Hospital Pharmacy is looking

for photographs for the cover of the Journal.

The photo theme for 2015 is:

\section{Canadian Landscapes and Scenery}

Interested participants are asked to submit a digital photo or group of photos for selection along with a short (max 150 words) write-up about the location of the photo, the date and time of the photo, and the type of equipment used. Be sure to include any stories or details that make this photograph or location unique! 